PROCEEDINGS OF THE

AMERICAN MATHEMATICAL SOCIETY

Volume 139, Number 1, January 2011, Pages 305-308

S 0002-9939(2010)10521-X

Article electronically published on August 5, 2010

\title{
SPHERICAL POINTS IN RIEMANNIAN MANIFOLDS
}

\author{
BENJAMIN SCHMIDT
}

(Communicated by Jon G. Wolfson)

\begin{abstract}
A point $p$ in a Riemannian manifold $M$ is weakly spherical if for each point $q \neq p$ there is either exactly one or at least three minimizing geodesic segments joining $p$ to $q$. In this note, it is shown that round 2dimensional spheres are the only Riemannian surfaces with a weakly spherical point realizing the injectivity radius.
\end{abstract}

Götz and Rybarski asked whether round spheres are the only convex surfaces with the property that every pair of points is joined either by a unique minimizing geodesic or by infinitely many minimizing geodesics [5, 4]. Zamfirescu answered this question affirmatively in 14 for $C^{3}$-smooth convex surfaces. In this note, round spheres are similarly characterized amongst all smooth Riemannian surfaces.

Definition 1. Let $M$ denote a closed Riemannian manifold. A point $p \in M$ is defined to be weakly spherical if for each distinct point $q \in M$ the set of minimizing geodesics joining $p$ to $q$ has either one element or at least three elements. A point $p \in M$ is defined to be strongly spherical if for each distinct point $q \in M$ the set of minimizing geodesics joining $p$ to $q$ has either one element or infinitely many elements.

Every point in a constant curvature sphere is strongly spherical. More generally, every point in a product of simply connected compact rank one symmetric spaces is strongly spherical. This raises the following question.

Question. Assume that $M$ is an irreducible closed Riemannian manifold with all points (strongly) spherical. Is $M$ isometric to a simply connected compact rank one symmetric space?

When $\operatorname{dim}(M)=2$ the answer is yes as proved below. Prior to stating this theorem, some notation is needed. Readers are referred to [3] for basic results about Riemannian manifolds.

Let $M$ denote a closed Riemannian manifold and $d$ its distance function. For $p \in M$, let $T_{p} M$ denote the tangent space to $p$ and $\operatorname{TCut}(p) \subset T_{p} M$ the tangent cut locus of $p$. Its image under the exponential map $\exp _{p}$ is the cut locus of $p$, denoted by $\operatorname{Cut}(p)$. The injectivity radius of $M$ at $p$ is defined by $\operatorname{inj}(p)=d(p, \operatorname{Cut}(p))$. The injectivity radius of $M$ is defined by $\operatorname{inj}(M)=\inf _{p \in M}\{\operatorname{inj}(p)\}$ and is realized by at least one point of $M$.

Received by the editors December 10, 2009 and, in revised form, March 30, 2010.

2010 Mathematics Subject Classification. Primary 53B21; Secondary 53C20, 53C22, 53C24, $53 \mathrm{C} 45$.

The author was supported in part by NSF Grant DMS-0905906.

(C)2010 American Mathematical Society 
Theorem 2. If $M$ is a closed Riemannian surface with a weakly spherical point realizing the injectivity radius, then $M$ is isometric to a constant curvature sphere.

Proof. Let $p \in M$ be a weakly spherical point of $M$. A point $q \in \operatorname{Cut}(p)$ is a cleave cut point if there are precisely two minimizing geodesic segments joining $p$ to $q$ such that $q$ is not conjugated to $p$ along either of these segments.

The 1-dimensional Hausdorff measure of non-cleave cut points in $\operatorname{Cut}(p)$ is zero [8. Proposition 2.1]. The hypothesis that $p$ is weakly spherical implies that no point in $\operatorname{Cut}(p)$ is a cleave cut point. Therefore, $\operatorname{Cut}(p)$ has zero 1-dimensional Hausdorff measure.

By [10], $\operatorname{Cut}(p)$ is a local tree (see [8, Theorem 2.3] for detailed definitions). A local tree with zero 1-dimensional Hausdorff measure is a single point. The conclusion of the theorem is now a consequence of the following lemma.

Lemma 3. Assume that $M$ is a closed Riemannian manifold and that $p \in M$ satisfies $\operatorname{inj}(p)=\operatorname{inj}(M)$. If $\operatorname{Cut}(p)$ is a single point, then $M$ is isometric to a constant curvature sphere.

Let $\operatorname{Cut}(p)=\{q\}$ and note that $M$ is homeomorphic to a sphere. Let $\operatorname{diam}(M)$ denote the diameter of $M$. By the resolution of the Blaschke conjecture for spheres 1, 9, 12, 13, metrics on $M$ with $\operatorname{inj}(M)=\operatorname{diam}(M)$ have constant curvature. It suffices to show that $\operatorname{diam}(M) \leq \operatorname{inj}(M) \operatorname{since} \operatorname{inj}(M) \leq \operatorname{diam}(M)$ always holds.

Choose points $x, y \in M$ such that $d(x, y)=\operatorname{diam}(M)$. The hypotheses imply that $x$ and $y$ lie in geodesics $\gamma_{x}$ and $\gamma_{y}$ of length $\operatorname{inj}(M)$ which join $p$ to $q$.

If $\gamma_{x}=\gamma_{y}$, then $\operatorname{diam}(M)=d(x, y) \leq \operatorname{inj}(M)$, concluding the proof in this case. Otherwise, $\gamma_{x} \cup \gamma_{y} \subset M$ is an embedded circle in $M$ of length $2 \operatorname{inj}(M)$. It follows easily that $\operatorname{diam}(M)=d(x, y) \leq \operatorname{inj}(M)$, concluding the proof of the lemma.

The final few results of this note begin to address the Question when $M$ has dimension greater than two.

Proposition 4. If $M$ is a closed Riemannian manifold with all points weakly spherical, then $M$ is simply connected.

Proof. The proof is by contradiction. If $M$ is not simply connected, then there is a closed curve $\gamma \subset M$ of minimal length amongst homotopically non-trivial closed curves in $M$. Necessarily, $\gamma$ is a closed geodesic. Fix $p \in \gamma$ and let $p^{\prime} \in \gamma$ denote its antipode. Then $\gamma$ consists of two geodesic segments joining $p$ to $p^{\prime}$, each of which is half the length of $\gamma$. Denote these geodesics by $\gamma_{0}$ and $\gamma_{1}$.

Claim. Both $\gamma_{0}$ and $\gamma_{1}$ are minimizing.

If not, then without loss of generality, $\gamma_{0}$ hits a cut point before arriving at $p^{\prime}$. Let $x$ be a point lying in $\gamma_{0}$ between this cut point and $p^{\prime}$ and let $\gamma_{2}$ be a minimizing geodesic joining $p$ to $x$. Then the closed curve formed by traversing $\gamma_{1}$ from $p$ to $p^{\prime}$, then $\gamma_{0}$ backwards from $p^{\prime}$ to $x$, and finally $\gamma_{2}$ backwards from $x$ to $p$ has length less than $\gamma$. By the choice of $\gamma$, this closed curve is homotopically trivial. It follows easily that the closed curve formed by traversing $\gamma_{0}$ from $p$ to $x$ and then $\gamma_{2}$ backwards from $x$ to $p$ is homotopic to $\gamma$. On the other hand, this curve has length less than $\gamma$. This contradiction concludes the proof of the claim.

The assumption that $p$ is weakly spherical implies that there exists a third minimizing geodesic $\gamma_{3}$ joining $p$ to $p^{\prime}$. If $\gamma_{0}$ is homotopic to $\gamma_{3}$ fixing endpoints, then the closed curve consisting of $\gamma_{3}$ followed by $\gamma_{1}$ backwards is homotopic to $\gamma$ and 
has equal length. However, this curve is not smooth at $p^{\prime}$ and can therefore be shortened in its free homotopy class, contradicting the choice of $\gamma$. Therefore, $\gamma_{0}$ and $\gamma_{3}$ are not homotopic fixing endpoints. It follows that the closed curve consisting of $\gamma_{0}$ followed by $\gamma_{3}$ backwards is homotopically non-trivial and has length equal to $\gamma$. On the other hand, this curve is not smooth at $p^{\prime}$ and can therefore be shortened in its free homotopy class. This contradiction completes the proof of the proposition.

Additional notation is needed for the final two results. For a point $p$ in a closed Riemannian manfold $M$, let $\operatorname{TConj}(p) \subset T_{p} M$ denote the locus of all conjugate vectors. The locus of first conjugate vectors will be denoted by

$$
\operatorname{FConj}(p)=\{v \in \operatorname{TConj}(p) \mid(0,1) v \cap \operatorname{TConj}(p)=\emptyset\} .
$$

A conjugate vector $v \in \operatorname{TConj}(p)$ is defined to be regular if there is a neighborhood $U$ of $v$ in $T_{p} M$ such that for each $u \in U$, the ray $[0, \infty) u \operatorname{intersects} \operatorname{TConj}(p) \cap U$ in a single point. When $\operatorname{TConj}(p)$ is non-empty, the locus of regular conjugate vectors forms a smooth codimension one submanifold of $T_{p} M$ that is relatively open and dense in $\operatorname{TConj}(p)$ [11, Theorem 3.1]. Moreover, the multiplicity of conjugate vectors is constant in each connected component of the regular conjugate locus.

Lemma 5. If $M$ is a closed Riemannian manifold and $p \in M$ is strongly spherical, then $\operatorname{FConj}(p)=\operatorname{TCut}(p)$. Moreover, $M$ is simply connected.

Proof. The second conclusion of the lemma is implied by the first (cf. the remark following [6, Lemma 2.2, pg. 19]). To prove the first statement of the lemma, it suffices to prove that each point $q \in \operatorname{Cut}(p)$ is conjugated to $p$ along some minimizing geodesic [7, Corollary 3.5].

Choose a vector $v \in \operatorname{TCut}(p)$ with $\exp _{p}(v)=q$. If $v \in \operatorname{FConj}(p)$, then $q$ is conjugated to $p$ along the minimizing geodesic $\eta(t)=\exp _{p}(t v)$. Otherwise, there is a vector $v^{\prime} \in \operatorname{TCut}(p) \backslash\{v\}$ with $\exp _{p}(v)=\exp _{p}\left(v^{\prime}\right)$. Since $p$ is strongly spherical, it follows that $X=\exp _{p}^{-1}(q) \cap \operatorname{TCut}(p)$ is a closed infinite set. Let $x \in X$ be an accumulation point of $X$. Since $\exp _{p}$ is not locally injective in a neighborhood of $x$, the inverse function theorem implies that $x \in \operatorname{TConj}(p)$. Since $\exp _{p}(x)=q$, the point $q$ is conjugated to $p$ along the minimizing geodesic $\gamma(t)=\exp _{p}(t x)$, concluding the proof.

Corollary 6. Let $M$ be a closed 3-manifold with a strongly spherical point $p \in M$ realizing the injectivity radius. If $\mathrm{FConj}(p)$ consists of regular conjugate vectors, then $M$ is isometric to a constant curvature sphere.

Proof. It suffices to prove that $\operatorname{Cut}(p)$ is a single point by Lemma 3 . Lemma 5 implies that $\operatorname{FConj}(p)=\operatorname{TCut}(p)$, which by hypothesis consists of regular conjugate vectors. In particular, $S:=\operatorname{FConj}(p)=\operatorname{TCut}(p)$ is a smooth 2-sphere consisting of conjugate vectors with a common multiplicity.

If the vectors in $S$ all have multiplicity one, then the kernels of the derivative maps of $\exp _{p}$ at these vectors defines a non-vanishing line field on $S$ by the proof of [6. Lemma 2.2, pg. 19]. This is a contradiction since $S$ is a smooth 2-sphere. Therefore, all vectors in $S$ have multiplicity two as conjugate vectors. The discussion immediately following [11, Theorem 3.2] implies that $\exp _{p}$ is locally constant on $S$. As $S$ is connected, $\operatorname{Cut}(p)=\exp _{p}(S)$ is a single point, concluding the proof. 


\section{REFERENCES}

1. M. Berger, Blaschke's conjecture for spheres, Appendix D in 2]. MR496885 (80c:53044)

2. A. L. Besse, Manifolds all of whose geodesics are closed, Springer-Verlag, Berlin-HeidelbergNew York, 1978. MR496885 (80c:53044)

3. J. Cheeger and D. Ebin, Comparison theorems in Riemannian geometry, North-Holland Mathematical Library, Vol. 9, 1975. MR0458335 (56:16538)

4. H. Croft, K. Falconer, and R. Guy, Unsolved problems in geometry, Springer-Verlag, New York, 1991. MR1107516 (92c:52001)

5. A. Götz and A. Rybarski, Problem 102, Colloquium Mathematicum 2 (1951), 301-302.

6. J. Hebda, Conjugate and cut loci and the Cartan-Ambrose-Hicks theorem, Indiana Math. J. 31, no. 1 (1982), 17-26. MR642612 (83j:53032)

7. J. Hebda, The local homology of cut loci in Riemannian manifolds, Tôhoku Math. Journ., 35 (1983), 45-52. MR695658 (84g:53066)

8. J. Hebda, Metric structure of cut loci in surfaces and Ambrose's problem, J. Diff. Geom. 40 (1994), 621-642. . MR.1305983 (95m:53046)

9. J. Kazdan, An inequality arising in geometry, Appendix E in 2]. MR496885 (80c:53044)

10. S. B. Myers, Connections between differential geometry and topology. II: Closed surfaces, Duke Math. J. 2 (1936), 95-102. MR1545908

11. F. Warner, The conjugate locus of a Riemannian manifold, Amer. J. Math. 87 (1965), 575604. MR0208534 (34:8344)

12. A. Weinstein, On the volume of manifolds all of whose geodesics are closed, J. Diff. Geom. 9 (1974), 513-517. MR 0390968 (52:11791)

13. C. T. Yang, Odd-dimensional wiedersehen manifolds are spheres, J. Diff. Geom. 15 (1980), no. 1, 91-96. MR602442 (82g:53049)

14. T. Zamfirescu, On some questions about convex surfaces, Math. Nachr. 172 (1995), 313-324. MR 1330637 (96e:52004)

Department of Mathematics, Michigan State University, East Lansing, Michigan 48824

E-mail address: schmidt@math.msu.edu 